\title{
Complexation stoechiometry determined by application of chemometrics to time domain nuclear magnetic resonance signals
}

\author{
C. Airiau, F. Gaudard, A.S. Barros and D.N. Rutledge* \\ Institut National Agronomique Paris-Grignon, Laboratoire de Chimie Analytique, 16 rue Claude Bernard, \\ 75231 Paris Cedex 05, France
}

\begin{abstract}
The objective of this study is to demonstrate the feasibility of using multivariate statistical techniques applied to Time Domain - Nuclear Magnetic Resonance (TD-NMR) signals to determine the stæchiometry of complexation reactions. The complex used here is the well known EDTA-manganese complex. The measurement is based on the change in the interactions between the paramagnetic manganese cation and the solvent depending on whether it is free in solution or chelated with EDTA. Free manganese significantly increases the relaxation rate of the solvating water protons while chelated manganese has a weaker effect. The complexation is studied using Job's Continuous Variations Method. The system is analysed in two ways. In a first step $R_{1}$ and $R_{2}$ relaxation rates are calculated by non-linear regression and are shown to be sensitive parameters for determining the stochiometry of the complex. In a second step, the normalised TD-NMR signals are directly analysed using Principal Components Analysis (PCA) and Evolving Factor Analysis (EFA). These multivariate statistical analyses of the signals are also shown to be good methods for detecting the complexation.
\end{abstract}

Key words. Time domain nuclear magnetic resonance - job's method - multivariate statistical analysis - complexation chemometrics.

\section{Introduction}

The object of this study is to demonstrate the feasibility of using Time Domain-Nuclear Magnetic Resonance (TDNMR) and multivariate statistical analysis techniques to determine the stœchiometry of complexations.

This type of reaction is currently characterised by spectral techniques such as Infra-Red [1], Ultra-Violet [13] or NMR spectroscopy $[9,11,12]$. On the other hand, although variations in the TD-NMR relaxation rates as a function of degree of complexation has been proposed as a method for the determination of proteins $[4,20]$, to to our knowledge this phenomenon has not yet been studied using chemometics. The TD-NMR technique is interesting from many point of view. It is non destructive and does not require the same specificity as U.V (being based on relaxation properties and not on the presence of chromophore groups). Furthermore, it requires a less expensive apparatus than does spectroscopic NMR.

In a first step, the analyses are performed on parameters calculated from TD-NMR signals: longitudinal $R_{1}$ and transverse $R_{2}$ relaxation rates. In a second step, multivariate statistical analyses are applied directly to the TD-NMR signals themselves.

To demonstrate the feasibility of using TD-NMR and multivariate statistical analysis in the study of stœchiometry of complexation reactions, we have chosen a very well characterised complex: manganese - EDTA (Ethylene Diamine TetraAcetate). Many studies $[2,12,18]$ have been carried out showing that EDTA and manganese cations form a complex with stœchiometry $1: 1$.
The basis of this study is the specific properties of paramagnetic compounds. Unpaired electrons in the external valence layer of a paramagnetic cation in solution in water create an electronic dipole/nuclear dipole interaction with the water protons. These interactions enhance the nuclear relaxation of the water leading to a significant increase in $R_{1}$ and $R_{2}$ values of the solution.

In this study, aqueous manganate solutions were used. The relaxivity of manganese (capacity to relax the solvent protons) will be different depending on whether the manganese is free and directly solvated by water or complexed by the ligand where the external electrons will be paired to some extent leading to a loss of part of the paramagnetic properties of the manganese cation. Furthermore, the ligands around the cations limit the direct contact with the water molecules. In other words, if the solutions contain free manganate, the $R_{1}$ and $R_{2}$ values should be very high whereas they will be much lower if the manganese cation is complexed by a ligand.

The ligand chosen to complex with manganese is EDTA (Ethylene Diamine Tetra Acetic acid): $\left(\mathrm{HOOCCH}_{2}\right)_{2} \mathrm{~N}-\mathrm{CH}_{2}-$ $\mathrm{CH}_{2}-\mathrm{N}\left(\mathrm{CH}_{2}-\mathrm{COOH}\right)_{2}$. In fact, this molecule has been studied very often and is known to complex 62 different metal cations with a $1: 1$ stœchiometry $[12,18]$. Its dissociation constant with manganese is $10^{-13} \mathrm{~mol}^{-1}[5]$.

To avoid any possible formation of $\mathrm{Mn}(\mathrm{OH})_{2}$, solutions were maintained at constant $\mathrm{pH}$ of 4.4 by a buffer solution of potassium hydrogenophthalate/sodium hydroxide [6] giving the $\mathrm{H}_{2} \mathrm{Y}^{2-}$ form of the weak acid EDTA [18]. The EDTA 
used was in the disodic dihydrate form: $\mathrm{Na}_{2} \mathrm{H}_{2} \mathrm{Y}, 2 \mathrm{H}_{2} \mathrm{O}$ (Merck) while the $\mathrm{MnCl}_{2}$ was used as is (Merck).

\section{Materials and methods}

Time Domain NMR measurements were performed on a $20 \mathrm{MHz}$ NMR instrument (Minispec pc120, Bruker). To avoid the samples heating up to the magnet temperature $\left(40{ }^{\circ} \mathrm{C}\right)$, they were maintained at $20{ }^{\circ} \mathrm{C}$ using a cryostat (Ministat Huber) circulating a perfluorinated fluid (Fluorinert, 3M).

Two standard TD-NMR pulse sequences were used:

The Carr-Purcell-Meiboom-Gill (CPMG) sequence [17] produces a transverse relaxation curve free from the effects of magnetic field inhomogeneity and imperfect $180^{\circ}$ pulses. The sequence is given by:

$$
\begin{gathered}
90_{x}-\tau-\left(180_{y}^{\circ}-2 \tau-180_{y}^{\circ}-2 \tau-180_{y}^{\circ}-2 \tau\right. \\
\left.\quad-180_{y}^{\circ}-\tau-\text { measurement }-\tau\right)_{n}-R D
\end{gathered}
$$

4 tau values $(0.5,1,1.5,4 \mathrm{~ms})$ were used in order to get information on possible chemical exchange-diffusion effects as well as the energy exchanges between neighbouring spins. The 4 signals were acquired for the same total duration of $480 \mathrm{~ms}$ corresponding to respectively $n=240,120,80,30$ points. The repetition delay (RD) was set to $10 \mathrm{~s}$. and the enhancement was 3 (9 scans). The points in the signals produce a decreasing monoexponential curve which can be modelled using the following equation:

$$
M(t)=M_{\mathrm{o}} \times \exp \left\{-R_{2} \cdot t\right\} .
$$

Where: $M_{0}$ : the initial amplitude

$R_{2}$ : the transverse relaxation rate

$M(t)$ : the signal intensity at time $t$.

The Inversion-Recovery (I-R) pulse sequence [7] was used to observe the longitudinal relaxation. The sequence can be represented by:

$$
180_{x}^{\circ}-\tau_{\mathrm{var}}-90_{y}^{\circ}-\tau-\text { measurement }-R D
$$

30 different $\tau_{\mathrm{var}}$ values from $3 \mathrm{~ms}$ to $18000 \mathrm{~ms}$ were generated using the following function:

$$
\tau_{\mathrm{var}}=3 *(1.35)^{[n-1]}
$$

were $n$, the current data point, varies from 1 to 30 . The signal intensity was measured at $\tau=40 \mu$ s after the $90^{\circ}$ pulse, RD was set to $10 \mathrm{~s}$. and signal enhancement was 3 .

This relaxation curve gives information on interaction between spins and their environment.

The signal is an increasing exponential curve of the form:

$$
M(t)=M_{\mathrm{o}}\left[1-2 \cdot \exp \left\{-R_{1} \cdot t\right\}\right] .
$$

Where $R_{1}$ is the longitudinal relaxation rate.

The $M(t), R_{1}$ and $R_{2}$ values were calculated using a nonlinear regression program [19] based on the Marquardt algorithm [16].

The Principal Components Analyses (PCA) and Evolving Factor Analyses (EFA) were performed on the TD-NMR signals using a Borland $\mathrm{C}++$ program developed in the laboratory to perform multivariate analyses on spectral data and images. The program routines have been validated against Matlab $^{\circledR}$ procedures.
To determine the influence of complexation on the responses obtained by TD-NMR, three sets of solutions of eleven samples each were prepared. The first two sets contain only increasing concentrations of either manganese or EDTA in buffer, from 0 to $1 \mathrm{mM}$ with a steps of $0.1 \mathrm{mM}$. The third set is composed of mixtures of EDTA and manganese prepared according to Job's Continuous Variations Method [10,13]. The total metal + ligand concentration in the 11 solutions is constant at $1 \mathrm{mM}$ but the ratio metal/(metal + ligand) varies from 0 to 1 . The parameter measured is plotted as a function of the molar fraction of ligand in solution. Depending on the detection technique used to monitor the formation of the complex, the curves may contain a critical point (maximum, minimum, inflexion) at a metal/ligand ratio corresponding to the stœchiometric ratio of the complex. However, it should be noted that the Job method is only applicable when only one complex is formed in solution.

The 4 CPMG curves and the I-R curve are first normalised to a maximum intensity of 1 and then decomposed by non linear regression to give $4 R_{2}$ and $1 R_{1}$ value. 11 sets of $R_{2}$ and $R_{1}$ values are calculated for the 3 series of solutions.

Each group of (4 CPMG + 1 IR) curves are then concatenated to give a single compound curve for multivariate statistical analysis. 3 matrices are thus formed containing 11 row vectors of 500 points each.

The data sets were analysed by Principal Component Analysis (PCA) [15] and Evolving Factor Analysis (EFA) $[3,14]$.

The PCA loadings (contributions of initial variables to the Principal Components) highlight the parts of the signal containing the most variability. The scores (projections of the objects onto the Principal Components) can reveal similarities and differences between individuals and highlight groupings of objects.

EFA is applied to the transposed data matrix. It consists in a series of PCAs performed initially on the first three columns of the transposed matrix (i.e. on the variables of the first three samples), then on the first four columns etc. until all eleven columns of the matrix are included. The eigenvalue of the first Principal Components for each PCA is plotted as a function of the number of columns used. Each successive eigenvalue will reflect the increased variability due to the addition of a new object and will increase much more if the sample is significantly different from its predecessors.

This multivariate statistical technique can be applied in the Forward and Backward modes. In Forward EFA, a PCA is done on the first three columns then on the first four columns and so on. Whereas backward EFA starts the PCA on the last three columns then on the last four columns etc. In both cases the eigenvalues increase with the number of columns.

\section{Results and discussion}

\section{Univariate analysis of NMR parameters}

Figure 1 presents the evolution of the $R_{2}$ values for the different solutions. The values calculated for the $4 \mathrm{CPMG}$ 


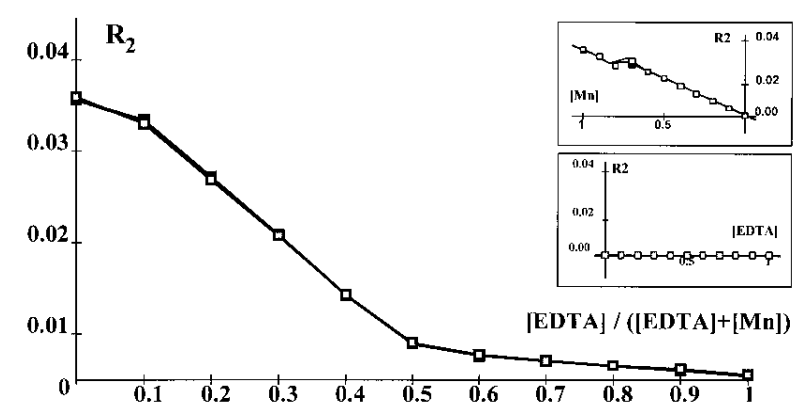

Fig. 1. $R_{2}\left(\mathrm{~m} \mathrm{~s}^{-1}\right)$ of EDTA-manganese solutions as a function of the molar fraction of EDTA (Top insert: $R_{2}$ of manganese; bottom insert: $R_{2}$ of EDTA).

curves are equal, within the limits of the errors associated within the non-linear decomposition, indicating that the $\tau$ value has no effect on the relaxation curve which means that diffusion - chemical exchange phenomena have no influence.

At $\mathrm{pH} 4.4$, proton exchange is acid-catalysed and the exchange rate so high that the interpulse delay has no visible effect on the $R_{2}$ values. The $R_{1}$ values are not plotted as they evolve in a very similar manner but are noisier.

For the EDTA solutions, the $R_{2}$ 's are low and almost constant. The $R_{2}$ 's of aqueous solutions of EDTA are not very different from that of water. The variation in EDTA concentration does not significantly influence the relaxation of the water protons.

For the manganate solutions, the variation in the $R_{2}$ relaxation rate is proportional to the analyte concentration in accordance with the expected effect of the paramagnetic manganate cations on the solvating water molecules.

As $R_{2}$ values are additives, it is possible to calculate the influence of the simultaneous presence of manganese cations and EDTA on the $R_{2}$ values. By subtracting the $R_{2}$ of the EDTA-manganese set from the sum of the corresponding $R_{2}$ 's of the manganese and the EDTA sets, it is possible to measure the effect of the complexed manganese in the EDTA-manganese set. Figure 2 presents the $R_{2}$ due to the presence of manganese bound into the EDTA-manganese complex.

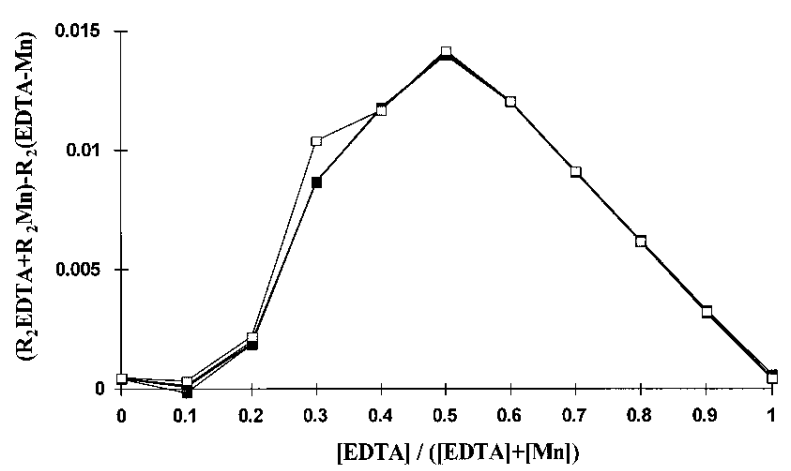

Fig. 2. Effect of complexation on $R_{2}$ values $\left(\mathrm{m} \mathrm{s}^{-1}\right)$.
The maximum corresponds to an equimolar solution, indicating a maximum complex concentration for this solution and thus a 1:1 complexation stœchiometry as expected.

\section{Multivariate statistical analyses}

Having demonstrated that TD-NMR can give information on complexations through the evolution of the calculated relaxation parameters, multivariate statistical analysis techniques were tested to see if it is possible to eliminate the non linear regression step and analyse the relaxation signals directly. Errors introduced by the non linear regression may be at the origin of the irregularities observed in figure 1 for the most concentrated manganese solutions which resulted in the Job curve (Fig. 2) being non-symmetrical.

\section{Principal component analysis}

A Principal Component Analysis was carried out on a column-centred data matrix composed of the normalised, concatenated relaxation curves of the three sets of solutions. The projection of the objects (solutions) onto the first two Principal Components is presented in figure 3. The eigenvalues indicate that $\mathrm{PC} 1$ and $\mathrm{PC} 2$ contain respectively $97.5 \%$ and $2.1 \%$ of the variability contained in the signals.

It is clear from figure 3 that all the EDTA solution are together except for the one with a concentration of $0.0 \mathrm{mM}$ (pure water) which overlaps the $0.0 \mathrm{mM}$ manganese solution. All other manganese solution are situated on the negative side of the PC1 axis and are distributed in order of increasing concentration.

The EDTA-Manganese solutions are also regularly distributed in order of increasing manganese concentration with the EDTA-Manganese 1:0 solution superimposed on the EDTA solutions and the EDTA-Manganese 0:1 solution almost on the top of the Mn $1.0 \mathrm{mM}$ solution. The equimolar EDTA-Mn solution is the last point on the negative side of the PC1 axis, almost on the top of the lowest concentration manganese solution. It is also the solution with the highest score on the PC2 axis. Solutions with excess EDTA are plotted on the positive side of PC1 and those with excess manganese on the negative side.

A plot of the scores onto the PC1-PC2 plane gives the same information as a standard Job plot based on a single parameter or measurement.

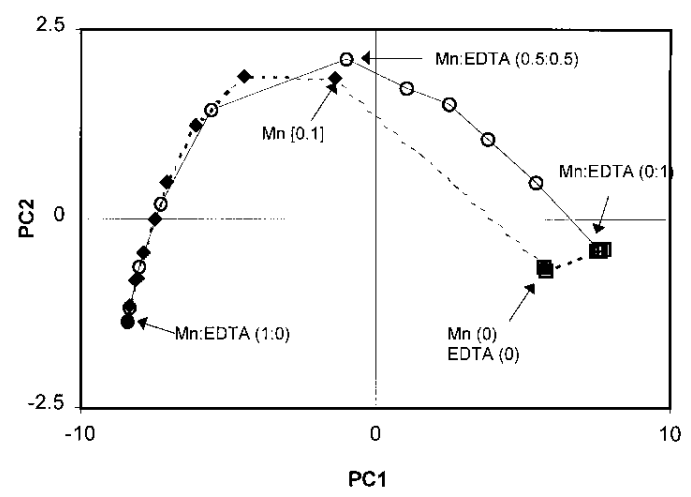

Fig. 3. Scores for the PCA applied to the three sets of solutions taken together. Manganese $(\diamond)$, EDTA $(\square)$, Mn_EDTA $(\bigcirc)$. 
The stœchiometry of the complexation can be clearly seen from the relative positions of the samples of the 3 sets of solution in the score plot. It is also clear that the complexed manganese has a significant influence on the relaxation of water as can be seen by the fact that the EDTA-manganese point with $0.2 \mathrm{mM}$ free $\mathrm{Mn}$ and $0.4 \mathrm{mM}$ complex is situated between $0.2 \mathrm{mM}$ and $0.3 \mathrm{mM}$ points in the Mn series. Similarly, the EDTA-manganese point with $0.4 \mathrm{mM}$ free manganese and $0.3 \mathrm{mM}$ complex is situated between the $0.4 \mathrm{mM}$ and $0.5 \mathrm{mM}$ manganese point.

All these results again confirm the 1:1 stœchiometry of the EDTA-manganese complex.

The plot of the Loadings for PC1 (Fig. 4) shows that the solutions are ordered along $\mathrm{PC} 1$ by the contribution of the final part of the CPMG curves. An increase in the quantity of slower relaxing components (i.e. low concentrations of manganese) displaces the sample towards the positive side of PC1. The negative first-derivative shape of the eigenvalues for the Inversion-Recovery curve indicates a shift in the Inversion-Recovery relaxation curve towards the right for the samples on the positive side of PC1. As for a monoexponential Inversion-Recovery curve the null-point corresponds to $\mathrm{T} 1 \times \log 2$, such a shift to the right would imply an increase in T1, which is to be expected when the Manganese concentration decreases.

The plot of the Loadings for PC2 shows that the solutions are ordered along PC2 by the contribution of the intermediate region of the CPMG curves. An increase in the quantity of these intermediate relaxing components (high concentrations of the Manganese_EDTA complex) displaces the sample towards the positive side of PC2. The secondderivative shape of the eigenvalues for the InversionRecovery indicates a shift in the null- point of the relaxation curve: both slower and faster relaxing components orient the samples towards the same side of PC2.

\section{Evolving factor analysis}

The second multivariate statistical analysis used is Forward and Backwards Evolving Factor Analysis [8] applied separately to each of the three data sets (Figs. 5-7).

Figures 5 and 6 present the calculated eigenvalues for the Forwards and Backwards EFA applied to the EDTA set and

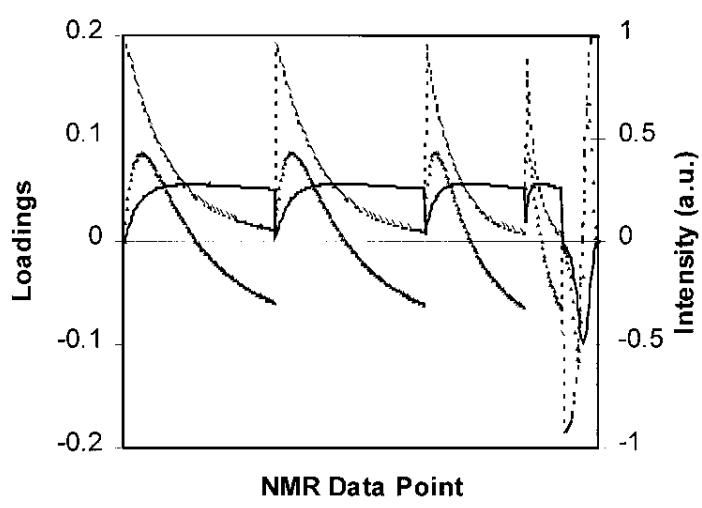

Fig. 4. Original signal (- -) and Loadings on PC1 (-) and on PC2 $(\boldsymbol{\Delta})$ for the PCA applied to the three sets of solutions. the manganese set, respectively. There is little variation between solutions as a function of concentration. The eigenvalues increase regularly as new columns are added due to the resulting increase in total variability of the data set. The eigenvalues for Manganese are much higher than those of EDTA as the addition of the cation has a greater effect on the relaxation properties of the solution.

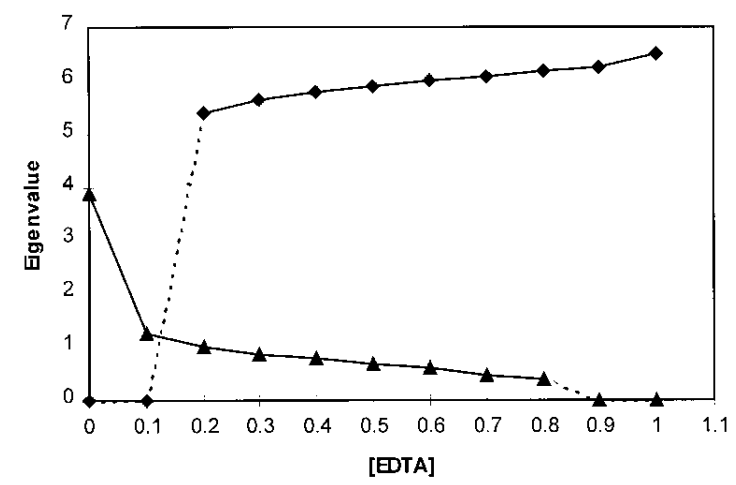

Fig. 5. Eigenvalues of first Principal Component for Forwards $(\bullet)$ and Backwards ( $\mathbf{\Delta})$ EFA on EDTA solutions as a function of EDTA molar ratio of last solution added.

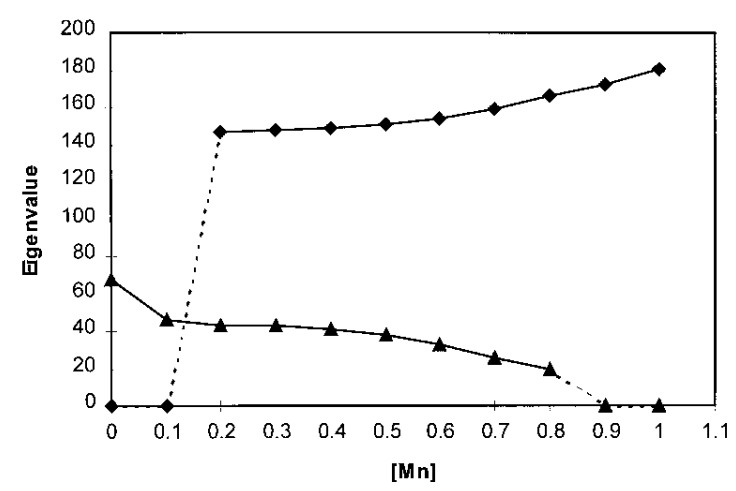

Fig. 6. Eigenvalues of first Principal Component for Forwards ( and Backwards $(\boldsymbol{\Lambda})$ EFA on Manganese solutions as a function of Manganese molar ratio of last solution added.



Fig. 7. Eigenvalues of first Principal Component for Forwards ( and Backwards ( $\mathbf{\Delta})$ EFA on Manganese_EDTA solutions as a function of EDTA molar ratio of last solution added. 
Figure 7 presents the eigenvalues of the Forward and Backward EFA on the Manganese_EDTA set. Here, there are even greater variations in the eigenvalues and these variations present a plateau in the region of the equimolar solution. Furthermore, the two series of eigenvalues cross at the level of this equimolar solution. This crossing over is characteristic of a data set in which a new species is being formed, and confirms the results obtained by PCA and the plotting of the $R_{2}$ values that the stœchiometry of the complexation is $1: 1$.

\section{Conclusion}

This study has demonstrated that Time Domain-NMR relaxation parameters may be used to determine complexation stœchiometry when one of the species in the complex, usually the metallic cation, has a significant influence on the relaxation rate of the solvent protons.

It has also shown that multivariate statistical techniques such as Principal Components Analysis and Evolving Factor Analysis can be directly applied to relaxation curves in order to determine the stœchiometry.

Further work is under way to determine to what extent the multivariate statistical analysis of TD-NMR signals may be applied with success to reactions other than metal-ligand complexations.

\section{References}

1. Alam, M. K.; Callis, J. B. Anal. Chem. 1994, 66, 2293-2300.

2. Belcher, R.; Gibbons, D.; West, T. S. Anal. Chim. Acta, 1955, 13, 226-229.
3. Brereton, R. G. Analyst 1995, 120, 2313-2336.

4. Coles, B. A. JAOCS 57(7) 202-204.

5. Charlot, G. L'analyse quantitative et les réactions en solution. Masson, Paris, 1963; pp 254-258.

6. C.R.C Handbook of Chemistry \& Physics, $62^{\text {nd }}$ Edition, CRC Press, Inc. Boca Raton, Florida, 1981-1982; D-126.

7. Farrar, T. C.; Becker, E. D. Pulse and Fourier transform NMR. Academic Press, 1971.

8. Gampp, H.; Maeder, M; Meyer, C. J., Zuberbuhler, A. D. Talanta 1986, 33(12), 943-951.

9. Godinez, L. A.; Lin, J.; Munoz, M.; Coleman, A. W.; Kaifer, A. E. J. Chem. Soc. 1996, 92, 645-650.

10. Hill, D. Z.; Mac Carthy, P. J. Chem. Educ. 1986, 63, 162-167.

11. Inoue, M. B.; Oram, P.; Inoue, M.; Fernado, Q. Inorg. Chim. Acta 1995, 232, 91-98.

12. Jen Fou Jen; Chih Shih Chen Anal. Chim. Acta 1992, 270, $55-61$.

13. Likussar, W.; Boltz, D. F. Anal. Chem. 1971, 43, 1265-1272.

14. Malinowski, E. R.; Howery, D. G. Factor Analysis in chemistry. Wiley \& Sons, New York, 1980.

15. Mardia, K. V., Kent, J. T.; Bibby, J. M. Multivariate analysis. Academic Press, London, 1995; pp 213-254.

16. Marquardt, D. W. J. Soc. Ind. Appl. Math. 1963, 11, 431-441.

17. Meiboom, S.; Gill, M. Rev. Sci. Instrum. 1958, 29, 688.

18. Skoog, D. M.; West, D. A. Fundamentals of Analytical Chemistry $2^{\text {nd }}$ Edition. A Holt International Edition, 1970; pp 343-357.

19. Rutledge, D. N. Data Handling in Science and Technology. Elsevier, Paris, 1996.

20. Wright, R. G.; Milward, R. C.; Coles, B. A. Food Technology 1980, 47-52. 